\title{
Antibiotic susceptibility of Brachyspira hyodysenteriae isolates from Czech swine farms: a 10-year follow-up study
}

\author{
Josef Prášek ${ }^{1}$, Daniel Šperling ${ }^{2}$, Dana Lobová ${ }^{1}$, Jiří Smola², Alois Čížek ${ }^{1}$
}

${ }^{1}$ University of Veterinary and Pharmaceutical Sciences, Faculty of Veterinary Medicine, Institute of Infectious
Diseases and Microbiology, ${ }^{2}$ Ruminant and Swine Clinic, Brno, Czech Republic

Received June 10, 2013

Accepted September 26, 2013

\begin{abstract}
Brachyspira hyodysenteriae is the causative agent of swine dysentery. Loss of clinical efficacy of some antimicrobial agents authorized for treating swine dysentery was observed on certain Czech pig farms. The aim of the present study was to evaluate the antimicrobial sensitivity of six antibiotics using a set of 202 randomly selected B. hyodysenteriae isolates obtained from farms in the Czech Republic between years 1997 and 2006. Minimum inhibitory concentration of antibiotics tylosin, lincomycin, tylvalosin, chlortetracyclin, tiamulin and valnemulin were tested, using an agar dilution method. All antibiotics tested showed an increase in minimal inhibitory concentrations. Continual decrease in susceptibility of B. hyodysenteriae isolates to tiamulin and valnemulin was observed. Multiresistant $B$. hyodysenteriae were isolated more frequently in the past years. Only a careful use of antibiotics can ensure their efficacy, especially in case of pleuromutilins, in the strategic therapy of swine dysentery. This rare study demonstrates the minimal inhibitory concentration changes of selected antidysenterics among Czech isolates of Brachyspira hyodysenteriae during a ten-year period.
\end{abstract}

Pigs, swine dysentery, therapy, minimal inhibition concentration, antimicrobial resistance

Brachyspira hyodysenteriae is an intestinal spirochete which colonizes the large intestine of pigs after being ingested, and induces diarrhoeal disease-swine dysentery (SD) (Hampson et al. 2006) which is spread worldwide. For the therapy and control of swine dysentery various antimicrobials have been used. The use of antimicrobials has been reduced in the past decade both due to a ban on nitroimidazoles and olaquindox, and the development of an acquired resistance of $B$. hyodysenteriae against formerly used antibiotics such as tylosin and lincomycin (Čížek et al. 2002). The situation resulted in an increased occurrence of clinical SD cases and spreading of the disease to other pig farms due to uncontrolled pig transports. Because of the gradual reduction of clinical efficacy of drugs of choice on swine farms with SD, the agent isolation and laboratory in vitro sensitivity tests were increasingly required. Pleuromutilins (tiamulin and valnemulin) became the last choice antibiotics for SD therapy on most affected farms. In the late 1990s, an increased use of pleuromutilins resulted in the rise of minimal inhibitory concentration (MIC) values (Lobová et al. 2004). Afterwards, a complete loss of clinical efficacy of one but sometimes even of both pleuromutilins was observed on some farms. When $B$. hyodysenteriae lost its sensitivity to most registered antimicrobials, there was a risk of spreading multiresistant clones of $B$. hyodysenteriae to other farms within integrations or even outside them.

The aim of the study was to evaluate the MICs for six antibiotics most frequently used for the treatment of SD, using a set of randomly selected Brachyspira hyodysenteriae isolated from pigs in the Czech Republic during years 1997 and 2006.

\section{Materials and Methods}

Bacterial isolates and growth conditions

In the period 1997-2006, the diagnostic service of the Institute of Infectious Diseases and Microbiology, UVPS Brno obtained 2035 isolates of Brachyspira hyodysenteriae. Of those, 202 were randomly selected for

Address for correspondence:

Josef Prášek

Institute of Infectious Diseases and Microbiology, Faculty of Veterinary Medicine

University of Veterinary and Pharmaceutical Sciences

Palackého tř. 1/3, 61242 Brno, Czech Republic
Phone: +420775956986

Fax: +420 541562274

E-mail: josef.prasek@gmail.com

http://actavet.vfu.cz/ 
the 10-year follow-up study. Only one isolate per farm and year was chosen. Isolates of B. hyodysenteriae were confirmed by the assessment of strong haemolysis, microscopy and species specific PCR (La et al. 2003). The cultures were stored at $-80{ }^{\circ} \mathrm{C}$ in cryoprotective medium. For susceptibility testing, thawed isolates were grown on Trypticase Soy agar (TSA) (BD BBL, USA) with 5\% ovine blood for up to 3-4 days in anaerobic jars (Oxoid, UK) with AnaeroGen sachets (Oxoid, UK) at $37{ }^{\circ} \mathrm{C}$. The purity of all cultures was confirmed by microscopy of crystal violet stained smears.

Control strains of Brachyspira hyodysenteriae B78 (ATCC 27164 ), Streptococcus pneumoniae ATCC 49619 and Staphylococcus aureus ATCC 29213 were included in the analyses as controls. Their MICs for pleuromutilin were published by Odland et al. (2000) and Karlss on et al. (2003).

\section{Susceptibility testing}

The antimicrobial susceptibility of Brachyspira hyodysenteriae isolates was determined by the agar dilution method based on Clinical and Laboratory Standards Institute (CLSI) guidelines for susceptibility testing of anaerobic bacteria (CLSI 2004). Wilkins-Chalgren anaerobe agar (CM 619, Oxoid) with 5\% ovine blood (WCABA) was used to determine MICs. The antimicrobial agents tested were tylosin (Elanco, USA), acetylisovaleryltylosin (Eco, UK), lincomycin (Sigma, Czech Republic), chlortetracycline (Sigma, Czech Republic), tiamulin fumarate and valnemulin (Novartis $\mathrm{AH}$, Switzerland). The concentrations of tested antibiotics were $0.031,0.062,0.125,0.25,0.5,1,2,4,8$ and $16 \mu \mathrm{g} / \mathrm{ml}$ of tylosin, lincomycin, chlortetracyklin, tiamulin and valnemulin in this order. The concentrations of acetylisovaleryltylosin were 3.125, 6.25,12.5, 25, 50, 100, $200 \mu \mathrm{g} / \mathrm{ml}$.

Pure Brachyspira hyodysenteriae cultures were scraped from Trypticase Soy agar with sterile cotton swabs and suspended in $2 \mathrm{ml}$ sterile phosphate buffered saline. The turbidity was adjusted with a photometer (Densi-La-Meter, LIAP, Latvia) to 1.0 McFarland standard. The suspension was added to the wells of a sterile microplate (Piove di Sacco, Italy) and multipoint inoculator (Trios, Czech Republic) was used to inoculate the agar surface. The final inoculum $(7-10 \mu \mathrm{l})$ on the agar surface provided approximately $10^{5} \mathrm{CFU}$ per spot. After 3-4 day incubation at $37{ }^{\circ} \mathrm{C}$, the result was read as MIC, i.e. the lowest concentration of the drug tested that prevented growth and haemolysis of the isolate on the inoculated spot. The growth of $B$. hyodysenteriae isolates was evaluated in parallel with controls on WCABA with no antimicrobial agents added. Three independent examinations of each of the $B$. hyodysenteriae isolates were made on different occasions with this test procedure.

\section{Statistical analysis}

Recorded MIC values were processed using MS Excel ${ }^{\circledR}$. When a difference of one dilution was found in a set of three repeated tests, the value that was obtained twice was used for further computations. In the case of a two-dilution difference, the mean value was used. Results with differences greater than two dilutions were not evaluated, and repeated MIC tests were made. The new results were evaluated in the same way. The values obtained for each of the drugs tested were used for the computation of $\mathrm{MIC}_{50}, \mathrm{MIC}_{90}$ and the range of $\mathrm{MICs}$.

\section{Results}

The determined minimal inhibitory concentrations of the six tested antibiotics for 202 Brachyspira hyodyseteriae isolates over the investigation period are presented in Table 1. Results confirmed that $\mathrm{MIC}$ values $\left(\mathrm{MIC}_{50}, \mathrm{MIC}_{90}\right)$ for tylosin and lincomycin were 64 to $128 \mu \mathrm{g} / \mathrm{ml}$ throughout the monitored period. A moderate MIC increase was observed in chlortetracycline and tylvalosin (acetylisovaleryltylosin) which was registered for pig treatment in the Czech Republic in 2000. An occurrence of B. hyodysenteriae with a high MIC value $(\geq 16 \mu \mathrm{g} / \mathrm{ml})$ to both the pleuromutilins at the same time was noted only in 2000 .

The percentage of Brachyspira hyodysenteriae isolates resistant to selected antibiotics is given in Table 2. Our results have shown that the percentage of isolates resistant to tiamulin and valnemulin was gradually increasing since 2000 . The percentage of tiamulin resistance was in total $24.3 \%$, rising from $22.2 \%$ in 2000 to $42.8 \%$ in $2005-2006$. Likewise valnemulin resistance was in total $33.2 \%$, rising from $37 \%$ in 2000 to $60.7 \%$ in $2005-2006$.

\section{Discussion}

Swine dysentery could have been controlled only by registered antimicrobials provided to larger groups of animals in the form of medicated feed or water, often at 
Table 1. Minimal inhibitory concentrations of six antimicrobials for $B$. hyodysenteriae isolates in the 10 -year period.

\begin{tabular}{|c|c|c|c|c|c|c|c|c|}
\hline \multirow[b]{2}{*}{$\begin{array}{l}\text { Antimicrobial } \\
\text { agent }\end{array}$} & \multicolumn{7}{|c|}{ Year of isolation and number of isolates in each year } & \multirow[b]{2}{*}{$\begin{array}{l}\text { Total } \\
(\mathrm{n}=202)\end{array}$} \\
\hline & $\begin{array}{c}1997-1998 \\
(\mathrm{n}=35)\end{array}$ & $\begin{array}{c}1999 \\
(n=26)\end{array}$ & $\begin{array}{c}2000 \\
(\mathrm{n}=27)\end{array}$ & $\begin{array}{l}2001 \\
(\mathrm{n}=38)\end{array}$ & $\begin{array}{r}2002-2003 \\
(\mathrm{n}=25)\end{array}$ & $\begin{array}{c}2004 \\
(n=23)\end{array}$ & $\begin{array}{c}2005-2006 \\
(\mathrm{n}=28)\end{array}$ & \\
\hline \multicolumn{9}{|l|}{ Tylosin } \\
\hline $\mathrm{MIC}_{50}$ & $>128^{*}$ & $>128$ & $>128$ & $>128$ & $>128$ & $>128$ & $>128$ & $>128$ \\
\hline $\mathrm{MIC}_{90}$ & $>128$ & $>128$ & $>128$ & $>128$ & $>128$ & $>128$ & $>128$ & $>128$ \\
\hline MIC range & $4->128$ & $8->128$ & $16->128$ & $2->128$ & $32->128$ & $4->128$ & $4->128$ & $4->128$ \\
\hline \multicolumn{9}{|l|}{ Tylvalosin } \\
\hline $\mathrm{MIC}_{50}$ & 12.5 & 25 & 25 & 50 & 50 & 50 & 50 & 25 \\
\hline $\mathrm{MIC}_{90}$ & 25 & 50 & 50 & 100 & 200 & 200 & 100 & 100 \\
\hline MIC range & $6.25-100$ & $3.125-100$ & $3.125-50$ & $3.125-100$ & $12.5-200$ & $3.125-200$ & $3.125-200$ & $3.125-200$ \\
\hline \multicolumn{9}{|l|}{ Lincomycin } \\
\hline $\mathrm{MIC}_{50}$ & 64 & 64 & 128 & 128 & 128 & 128 & 128 & 64 \\
\hline $\mathrm{MIC}_{90}$ & 64 & 128 & 128 & 128 & 128 & 128 & 128 & 128 \\
\hline MIC range & $4-128$ & $2-128$ & $4-128$ & $2-128$ & $4-128$ & $2-128$ & $2-128$ & $2-128$ \\
\hline \multicolumn{9}{|c|}{ Chlortetracyklin } \\
\hline $\mathrm{MIC}_{50}$ & 4 & 2 & 4 & 4 & 8 & 16 & 8 & 4 \\
\hline $\mathrm{MIC}_{90}$ & 8 & 8 & 8 & 4 & 16 & 32 & 8 & 16 \\
\hline MIC range & $1-16$ & $1-16$ & $1-8$ & $1-8$ & $1-32$ & $1-32$ & $1-8$ & $1-32$ \\
\hline \multicolumn{9}{|l|}{ Tiamulin } \\
\hline $\mathrm{MIC}_{50}$ & 0.125 & 0.125 & 2 & 2 & 4 & 1 & 4 & 0.5 \\
\hline $\mathrm{MIC}_{90}$ & 0.25 & 0.5 & 8 & 16 & 16 & 16 & 16 & 16 \\
\hline MIC range & $\leq 0.03-0.5$ & $\leq 0.03-8$ & $0.06-16$ & $\leq 0.03->16$ & $0.125->16$ & $0.06->16$ & $\leq 0.03->16$ & $\leq 0.03->16$ \\
\hline \multicolumn{9}{|l|}{ Valnemulin } \\
\hline $\mathrm{MIC}_{50}$ & $\leq 0.03$ & $\leq 0.03$ & 2 & 0.5 & 4 & 2 & 8 & 0.25 \\
\hline $\mathrm{MIC}_{90}$ & 0.06 & 0.5 & 8 & 16 & 16 & 16 & 16 & 16 \\
\hline MIC range & $\leq 0.03-0.5$ & $\leq 0.03-1.0$ & $\leq 0.03-16$ & $\leq 0.03->16$ & $\leq 0.03->16$ & $\leq 0.03->16$ & $\leq 0.03->16$ & $\leq 0.03->16$ \\
\hline
\end{tabular}

*MIC - minimal inhibitory concentrations, values are presented in $\mu \mathrm{g} / \mathrm{ml}$,

$\mathrm{MIC}_{50}, \mathrm{MIC}_{90}$ - Minimum Inhibitory Concentration required to inhibit the growth of $50 \%$ or $90 \%$ of tested isolates

Table 2. Comparative number of resistant isolates of B. hyodysenteriae in Czech swine farms during the 10-year period.

\begin{tabular}{|c|c|c|c|c|c|c|c|c|c|}
\hline \multirow[b]{2}{*}{$\begin{array}{l}\text { Antimicrobial } \\
\text { agents }\end{array}$} & \multicolumn{8}{|c|}{ Resistant isolates (\%) } & \multirow[b]{2}{*}{$\begin{array}{c}\text { Breakpoint* } \\
(\mu \mathrm{g} / \mathrm{ml})\end{array}$} \\
\hline & $\begin{array}{c}1997-1998 \\
(\mathrm{n}=35)\end{array}$ & $\begin{array}{c}1999 \\
(n=26)\end{array}$ & $\begin{array}{c}2000 \\
(\mathrm{n}=27)\end{array}$ & $\begin{array}{c}2001 \\
(\mathrm{n}=38)\end{array}$ & $\begin{array}{c}2002-2003 \\
(\mathrm{n}=25)\end{array}$ & $\begin{array}{c}2004 \\
(n=23)\end{array}$ & $\begin{array}{c}2005-2006 \\
(\mathrm{n}=28)\end{array}$ & $\begin{array}{l}\text { Total } \\
(\mathrm{n}=202)\end{array}$ & \\
\hline Tylosin & $34(97.1)$ & $26(100)$ & $27(100)$ & $36(94.7)$ & $25(100)$ & $22(95.6)$ & $27(96.4)$ & $198(98)$ & $\geq 4$ \\
\hline Tylvalosin & 0 & $4(15.4)$ & $6(22.2)$ & $20(52.6)$ & $17(68)$ & $15(65.2)$ & $18(64.3)$ & $83(41.3)$ & $\geq 32 * *$ \\
\hline Lincomycin & 24 (68.6) & $16(61.5)$ & 25 (92.6) & $35(92.1)$ & $23(92)$ & $19(82.6)$ & $23(82.1)$ & $165(81.7)$ & $\geq 36$ \\
\hline Tiamulin & 0 & $1(3.8)$ & $6(22.2)$ & $9(23.7)$ & $12(48)$ & $9(39.1)$ & $12(42.8)$ & $49(24.3)$ & $\geq 4$ \\
\hline Valnemulin & 0 & $1(3.8)$ & $10(37)$ & $15(39.5)$ & $13(52)$ & $11(47.8)$ & $17(60.7)$ & $67(33.2)$ & $\geq 4$ \\
\hline
\end{tabular}

* interpretative criteria according to clinical breakpoints (Rønne and Szancer 1990), ** clinical breakpoint according Burch (2005); breakpoint for chlortetracycline was not determined.

preventative dosage. In such cases an optimum relation between pharmacokinetics and pharmacodynamics of a drug could not be achieved and so called "selection window" that facilitates the development of antibiotic resistance was enlarged (McKellar et al. 2004). 
In our study, we found high MIC values of tylosin and linkomycin throughout the period under study. This is explained by the fact that both medicinal substances had been widely used for both preventative and therapeutical medication against SD on pig farms long before antibiotic growth promoters were banned. Decreased efficacy of the antibiotics in the therapy of SD was reported also in Sweden (Karlsson et al. 2003). It has been also found that point mutations that cause resistance against tylosin can be associated with a negligible increase in MIC of tylvalosin (Karlsson et al. 2004). Moderate MIC increase was observed in our study for tylvalosin (acetylisovaleryltylosin) which was registered for pigs in the Czech Republic in 2000. The isolates with increased MIC of tylvalosin were also obtained. This suggests that there are some other mechanisms of resistance development, so far unknown. For the assessment of Brachyspira hyodysenteriae sensitivity to tylvalosin a breakpoint $\geq 32 \mu \mathrm{g} / \mathrm{ml}$ reported by Burch (2005) was chosen.

The previous study confirmed the decreasing trend in sensitivity of isolates of B. hyodysenteriae to pleuromutilins in the Czech Republic (Lobová et al. 2004a,b). Unlike other EU countries, in the Czech Republic valnemulin had been used since 1999. Results of our study demonstrate a continual decrease in sensitivity of $B$. hyodysenteriae isolates to both pleuromutilins, i.e. tiamulin and valnemulin. The trend of decreasing sensitivity to tiamulin and valnemulin has been recently observed also in other countries.

Tiamulin resistance against Brachyspira hyodysenteriae develops gradually both in vitro and in vivo as a result of mutations in ribosomal protein L3 and 23S rRNA genes (Pringle et al. 2004). Consequently, MIC of tiamulin has been growing which is reflected as different levels of sensitivity to tiamulin in B. hyodysenteriae isolates (Karlsson et al. 2001; Karlsson et al. 2004). In clinical practice, frequent use of tiamulin causes selection pressure due to which the clones of $B$. hyodysenteriae with reduced sensitivity to tiamulin have prevailed in some herds. This is the reason why a wide range of tiamulin MIC values was found in the investigated set of $B$. hyodysenteriae isolates from different farms (Karlsson et al. 2004). In the set of isolates from many pig farms a broad range of tiamulin MIC values was found from 0.03 to $>16 \mu \mathrm{g} / \mathrm{ml}$.

Pringle et al. (2012) reported a moderate increase of tiamulin MIC. They found during investigations of Swedish isolates of Brachyspira hyodysenteriae in 1990-2003 that stopped tiamulin MIC increasing a few years ago as a result of implementation of the national swine dysentery eradication programme. In Poland the occurrence of resistance against tiamulin and valnemulin has not been confirmed so far (Zmudski et al. 2012).

Our results have shown that the percentage of isolates resistant to valnemulin has been higher than percentage of isolates resistant to tiamulin since 2000. Resistance to valnemulin had been found in the Czech Republic before valnemulin was registered for pigs. It is assumed that there might be a cross resistance between the two pleuromutilins (Lobová et al. 2004). An increased occurrence of Brachyspira hyodysenteriae with a high MIC value $(\geq 16 \mu \mathrm{g} / \mathrm{ml})$ to both the pleuromutilins at the same time was noted only in 2000 .

Swine dysentery as a permanently present infectious disease is treated and controlled by a group medication with selected antimicrobial substances. The risk of resistance to pleuromutilins may pose a serious threat for swine production in the near future. Therefore such antibiotics should be used only as a last resort and in programmes of SD eradication. Only prudent use of antibiotics can secure efficacy of antibiotics, pleuromutilins in particular, in the strategic therapy of swine dysentery.

\section{Acknowledgement}

This study was supported in part by the National Agency of Agricultural Research, Grant No. QI111A166. 


\section{References}

Burch DGS 2005: Pharmacokinetic, pharmacodynamic and clinical correlations relating to the therapy of colonic infections in the pig and breakpoint determinations. Pig J 56: 8-24

Čížek A, Lobová D, Smola, J 2002: In vitro susceptibility of Brachyspira hyodysenteriae strains isolated in the Czech Republic from 1996 to 2001. Proceedings of the $17^{\text {th }}$ International Pig Veterinary Society Congress. Ames, USA: p. 191

CLSI 2004: Methods of antimicrobial susceptibility testing of anaerobic bacteria. Clinical and Laboratory Standards Institute. Document M11-A6. Edited by CLSI, Wayne, Pennsylvania

Hampson DJ, Fellström C, Thomson J 2006: Swine dysentery. In: Straw BE, D'Allaire S, Mengeling WL, Taylor DJ, (Eds): Diseases of Swine. Blackwell Publishing, Oxford, UK, p. 785-805

Karlsson M, Aspan A, Landén A, Franklin A 2004: Futher characterisation of porcine Brachyspira hyodysenteriae isolates with decreased susceptibility to tiamulin. J Med Microbiol 53: 281-285

Karlsson M, Fellström C, Gunnarsson A, Landén A, Franklin A 2003: Antimicrobial susceptibility testing of porcine Brachyspira (Serpulina) species isolates. J Clin Microbiol 41: 2596-2604

Karlsson M, Gunnarsson A, Franklin A 2001: Susceptibility to pleuromutilins in Brachyspira (Serpulina) hyodysenteriae. Anim Health Res Rev 2: 59-65

La T, Phillips ND, Hampson DJ 2003: Development of a duplex PCR assay for detection of Brachyspira hyodysenteriae and Brachyspira pilosicoli in pig feces. J Clin Microbiol 41: 3372-3375

Lobová, D, Ćížek, A 2004a: In vitro assessment of susceptibility of doxycycline to Brachyspira hyodysenteriae using agar dilution method and Epsilometer test. Acta Vet Brno 73: 329-333

Lobová D, Smola J, Čížek J 2004b: Decreased susceptibility to tiamulin and valnemulin among Czech isolates of Brachyspira hyodysenteriae. J Med Microbiol 53: 287-291

McKellar QA, Sanchez Bruni SF, Jones DG 2004: Pharmacokinetic/pharmacodynamic relationships of antimicrobial drugs used in veterinary medicine. J Vet Pharmacol Therap 27: 503-514

Odland BA, Erwin ME, Jones RN 2000: Quality control guidelines for disk diffusion and broth microdilution antimicrobial susceptibility tests with seven drugs for veterinary applications. J Clin Microbiol 38: 453-455

Pringle M, Landén A, Unnerstad HE, Molander B, Bengtsson B 2012: Antimicrobial susceptibility of porcine Brachyspira hyodysenteriae and Brachyspira pilosicoli isolated in Sweden between 1990 and 2010. Acta Vet Scand 54: 54

Pringle M, Poehlsgaard J, Vester B, Long KS 2004: Mutations in ribosomal protein L3 and 23S ribosomal RNA at the peptidyl transferase centre are associated with reduce susceptibility to tiamulin in Brachyspira spp. isolates. Molecul Microbiol 54: 1295-1306

Rønne H, Szancer J 1990: In vitro susceptibility of Danish field isolates of Treponema hyodysenteriae to chemotherapeutics in swine dysentery (SD) therapy. Interpretation of MIC results based on the pharmacokinetic properties of the antibacterial agents. In Proc 11 ${ }^{\text {th }}$ Int Pig Vet Soc Congr, Lausanne, Switzerland: p. 1126

Zmudski J, Szczotka A, Nowak A, Strzelecka H, Grzesiak A, Pejsak Z 2012: Antimicrobial susceptibility of Brachyspira hyodysenteriae isolated from 21 Polish farms. Pol J Vet Sci 15: 259-265 
